\title{
Fistules obstétricales dans le district d'Abidjan, Côte d'Ivoire : niveau de connaissance et plantes utilisées traditionnellement dans le traitement
}

\author{
Stéphanie Marianne-Lébri LAGOU ${ }^{1}$, Fézan Honora TRA BI ${ }^{1}$, Konan YAO $^{2,3}$, \\ Adama BAKAYOKO ${ }^{1,3}$ et Mamidou Witabouna KONE ${ }^{1,3, *}$ \\ ${ }^{1}$ UFR Sciences de la Nature, Université Nangui Abrogoua, BP 801 Abidjan 02, Côte d'Ivoire. \\ ${ }^{2}$ UFR Biosciences, Université Félix Houphouët-Boigny, BP 582 Abidjan 22, Côte d'Ivoire. \\ ${ }^{3}$ Centre Suisse de Recherches Scientifiques en Côte d'Ivoire, BP 1303 Abidjan 01, Côte d'Ivoire. \\ *Auteur correspondant ; E-mail: konewit_sn@una.edu.ci; mamidou.kone@csrs.ci; Tel: + 22503488905.
}

\section{RESUME}

La fistule obstétricale est une lésion résultant d'un travail d'accouchement prolongé. Ce problème de santé est une complication courante qui afflige de nombreuses femmes en Afrique, notamment en Côte d'Ivoire. Cette étude a été menée pour déterminer le niveau de connaissance des femmes et la prise en charge traditionnelle de cette maladie. Une enquête a été menée dans trois quartiers (Yopougon, Abobo et Treichville) dans le district d'Abidjan et comprenait 560 femmes. Parmi ces femmes, 25,71\% ont déclaré avoir des informations sur cette pathologie. La fistule obstétricale peut être guérie (76.39\% des répondants) et la médecine moderne est la méthode la plus appropriée selon $81,73 \%$ des femmes. Certaines femmes $(18,27 \%)$ préfèrent la médecine traditionnelle. Une liste de 13 espèces végétales (13 genres et 8 familles) a été rapportée comme traditionnellement utilisée dans le traitement de la fistule obstétricale. Les familles des Lamiaceae, Meliaceae, Malvaceae et Euphorbiaceae étaient les plus représentatives. Ces plantes pourraient jouer un rôle dans la prise en charge de la fistule obstétricale.

(c) 2016 International Formulae Group. All rights reserved.

Mots clés : Fistule obstétricale, connaissance, ethnobotanique, traitements, District d'Abidjan, Côte d'Ivoire.

\section{Obstetric fistula in the district of Abidjan, Cote d'Ivoire: Knowledge and plants traditionally used for treatment}

\begin{abstract}
Obstetric fistula is an injury resulting from prolonged obstructed labour. This health problem is a common complication afflicting many women in Africa, particularly in Côte d'Ivoire. This study was carried out to determine the women's knowledge level and traditional management of this disease. A survey was conducted in three areas (Yopougon, Abobo and Treichville) in the district of Abidjan and included 560 women. Of these women, $25.71 \%$ stated they had some information about this disease. Obstetric fistula can be cured (76.39\% of respondents) and modern medicine is the most suitable method according $81.73 \%$ of women. Some women (18.27\%) preferred traditional medicine. A list of 13 plant species (13 genera and 8 families) was
\end{abstract}


reported as traditionally used in the treatment of obstetric fistula. The families of Lamiaceae, Meliaceae, Malvaceae and Euphorbiaceae were the most representative. These plants may play a role in the management of obstetric fistula.

(c) 2016 International Formulae Group. All rights reserved.

Keywords: Obstetric fistula, knowledge, ethnobotany, treatments, Abidjan, Côte d'Ivoire.

\section{INTRODUCTION}

La grossesse est l'une des causes majeures de la mortalité des femmes dans les pays pauvres (Milliez, 2009). Chaque année, 500.000 femmes meurent parce qu'elles sont enceintes (Anderson et al., 2007). La Côte d'Ivoire enregistre un taux de mortalité maternelle qui oscille entre 597 et 690 pour 100.000 naissances vivantes (OMS, 2004, 2010). Les facteurs qui peuvent expliquer ce niveau élevé de mortalité maternelle sont essentiellement le nombre important d'enfants par femme, les naissances rapprochées, mais surtout les accouchements sans assistance médicale.

L'accouchement sans assistance médicale peut avoir plusieurs conséquences tragiques. Les complications obstétricales sont très importantes chez les femmes des pays en développement. Dans ce lot de souffrances et invalidités permanentes émergent les fistules obstétricales.

La fistule obstétricale est une communication anormale entre les voies génitales et urinaires (Kouye et al., 2006). Elle est caractérisée par une perte permanente d'urines et/ou de selles, sans possibilité de contrôle. De toutes les infirmités liées à la grossesse, elle est celle qui laisse le plus de séquelles aux plans physique et moral. Cette pathologie a de lourds impacts individuels, sociaux et économiques. La fistule obstétricale reste la morbidité la moins prise en charge bien qu'elle soit celle qui affecte le plus la femme, la famille et la société. Si les pays développés ont réussi à éliminer les fistules obstétricales depuis près d'un siècle grâce à l'amélioration des conditions de vie et de santé en particulier l'accès aux Soins Obstétricaux d'Urgence, la situation est différente dans les pays en développement. Au niveau de ces pays, la fistule obstétricale est encore très prévalente, on estime la prévalence à 2000000 et l'incidence (nouveaux cas) entre 50.000 et 100.000 cas chaque année (UNPFA, 2003). Selon les statistiques de l'OMS, sur 1000 femmes décédées des suites d'un accouchement dans les pays en développement, 10 succombent à une fistule obstétricale (UNFPA, 2013). En Afrique subsaharienne, il existerait plusieurs millions de cas (Wall, 2006, 2012). En Côte d'Ivoire, la prévalence de cette pathologie est difficile à estimer à cause de la pauvreté de la documentation véritable sur le sujet, du caractère tabou de la maladie, des problèmes d'accessibilité des femmes malades aux rares centres de référence situés dans la capitale et surtout, du coût élevé du traitement. La chirurgie reste le seul moyen efficace de guérison proposé par la médecine moderne (Kouye et al., 2006). Par exemple, au Cameroun, 46,4\% des femmes victimes de fistules obstétricales interviewées $(n=28)$ ont subi une intervention chirurgicale réparatrice (Sanou et al., 2015). Cependant, cette chirurgie est l'une des interventions les plus difficiles, souvent émaillée de nombreux échecs entraînant de multiples hospitalisations et mettant ainsi, à rude épreuve, la patience de la malade (Xavier et al., 2007). 
L'objectif de ce travail est de déterminer le niveau de connaissance des femmes sur les fistules obstétricales et de recenser des plantes médicinales potentiellement utilisables dans le traitement dans le district d'Abidjan (Sud Côte d'Ivoire).

\section{MATERIEL ET METHODES}

\section{Zone d'étude}

Située au Sud de la Côte d'Ivoire, la ville d'Abidjan est la capitale économique du pays. Elle est bordée par le Golf de Guinée et traversée par la lagune Ebrié. Abidjan s'étend sur une superficie de $422 \mathrm{~km}^{2}$ et est constituée de 10 communes (Figure 1). Abobo, Yopougon et Treichville sont trois d'entre elles, et ont constitué la zone d'étude. La commune d'Abobo est située au Nord de l'agglomération d'Abidjan, et couvre une superficie de $78 \mathrm{~km}^{2}$. La commune de Treichville, quant à elle, couvre une superficie de $77,45 \mathrm{~km}^{2}$. Elle est localisée au Sud d'Abidjan (Kadjo, 2014). La commune de Yopougon est la plus grande commune de la capitale économique mais aussi du pays. Elle s'étend sur une superficie de 153,06 km² et est située à l'Ouest de la ville d'Abidjan (Bossart, 2006). Ces trois communes ont été choisies du fait qu'elles sont les plus peuplées de populations de niveau social moyen à faible.

\section{Echantillonnage}

L'unité d'échantillonnage est le menage. La taille de l'échantillon a été calculée selon la méthode décrite par l'OMS (1991) : $\quad \mathbf{n}=\mathbf{t}^{\mathbf{2}} \mathbf{x} \mathbf{p}(\mathbf{1}-\mathbf{p}) / \mathbf{m}^{\mathbf{2}}$

avec $\mathrm{n}=$ taille d'échantillon requise, $\mathrm{t}=1,96$ à un niveau de confiance de $95 \%$, $\mathrm{p}=$ prévalence estimative des maladies dans la zone d'étude et $\mathrm{m}=$ marge d'erreur à $4,14 \%$. Selon Bouvet et Grimont (2001), lorsque la prévalence d'une pathologie n'est pas connue, une prévalence de $50 \%$ est considérée afin d'obtenir une taille maximale d'échantillon.

Ainsi, un échantillon de 560 ménages à enquêter a été obtenu, reparti proportionnement à la taille de chacune des trois communes choisies $(250$ femmes à Abobo, 50 femmes à Treichville et 260 femmes à Yopougon).

\section{Etude descriptive et comparative}

Cette étude descriptive et comparative a été réalisée dans les communes d'Abobo, Treichville et de Yopougon de Mars 2015 à Juillet 2015. La population de l'étude est constituée de toute femme ayant au moins un enfant et habitant dans ces différentes communes. Les femmes ont été choisies selon la méthode de proche en proche (Blanchet et Gotman, 2007). Avant chaque interview, l'accord de chaque femme à se soumettre au questionnaire a été obtenu après les explications des objectifs de l'enquête.

Le questionnaire portait sur les caractéristiques sociodémographiques (âge, niveau d'étude, situation sociale, etc.) des femmes, leur niveau de connaissance des fistules obstétricales et enfin sur les attitudes pour le traitement de la maladie.

\section{Enquête ethnobotanique}

L'enquête ethnobotanique a été menée à l'aide des interviews semi-structurées à partir d'un questionnaire. Les questions ont été axées sur le nom local de la maladie, les organes de la plante utilisée, les indications thérapeutiques, les modes d'administration.

Les échantillons d'herbier ont été confectionnés et identifiés à l'Université Nangui Abrogoua, puis authentifiés au Centre National de Floristique (CNF) de l'Université Félix Houphouët-Boigny d'Abidjan. 


\section{Traitement des données}

- Fréquences de citation (FC)

La fréquence de citation de chaque espèce a été évaluée suivant la formule suivante :

$\mathbf{F C}=\mathbf{N P} / \mathbf{N T}$

avec NP: nombre de fois où l'espèce est citée ; NT : nombre total de citations

- $\quad$ Contribution de chaque espèce (Cpr)

La contribution de chaque plante dans la constitution des recettes (Cpr) a été également évaluée selon Dassou et al. (2014). Elle a permis de connaître la fréquence d'implication d'une plante dans les recettes et a été exprimée par la formule :

$\mathrm{Cpr}=(\mathrm{Nr} / \mathrm{Nt}) \times 100$

Avec $\mathrm{Nr}$ : nombre de recettes sollicitant la plante ; Nt : nombre total de recettes.

- Indice de confirmation ou Consensus d'informateurs (IC)

Le facteur ou degré de consensus d'utilisation ou Informant Consensus Factor (IC) est utile pour apprécier les accords des informateurs sur les thérapies rapportées. L'IC est calculé selon la formule : $\quad \mathbf{I C}=\mathbf{n}_{\mathbf{u r}}-\mathbf{n}_{\mathbf{t}} / \mathbf{n}_{\mathbf{u r}} \mathbf{- 1}$

$\mathrm{n}_{\mathrm{ur}}$ désigne le nombre de citation d'utilisation de chaque catégorie ;

$\mathrm{n}_{\mathrm{t}}$ le nombre total des espèces utilisées.

L'IC varie entre [0-1]. Une valeur faible, proche de 0 , indique que les informateurs sont en désaccord sur les thérapies proposées. Une valeur élevée, proche de 1 , indique un consensus élevé ou total autour de l'usage de la plante.

\section{Analyses statistiques}

La saisie des données a été réalisée avec le logiciel Epidata 3.1 et transférée sur le logiciel SPSS 20.0 pour la mise en place de la base de données. Le test de Khi-deux $\left(\chi^{2}\right)$ a été utilisé pour établir, s'il existe, une relation entre le niveau de connaissance des fistules obstétricales et des paramètres tels que le lieu d'habitation et le niveau d'instruction, à l'aide du logiciel SPSS 20.0. Lorsqu'une relation existe au seuil de $0,05 \%$, le test de Khi-deux est complété par la procédure de Marascuilo (Marascuilo et Serlin, 1988) à l'aide du logiciel X1Stat version 2014.5.03. Cette procédure consiste à effectuer des tests de comparaison deux à deux pour tous les couples de proportions, ce qui permet d'identifier les proportions responsables de l'existence de liaison. La plus petite différence significative a été fixée au seuil de 0,05 (Vessereau, 1992).

\section{RESULTATS}

Caractéristiques socio-démographiques des femmes interrogées

L'âge des femmes interrogées variait de 18 à plus de 45 ans, la tranche d'âge de 3045 est la plus représentée. Les femmes sont instruites pour la plupart avec des niveaux de formations allant du primaire au supérieur. Le nombre d'analphabètes n'est néanmoins pas négligeable. Elles exercent différentes activités professionnelles et sont mariées pour la plupart (Tableau 1).

\section{Niveau de connaissance des fistules obstétricales}

Sur les 560 femmes interrogées, $25,71 \%$ ont une fois entendu parler des fistules obstétricales et $74,29 \%$ ne savent rien de la pathologie (Tableau 2). Parmi les femmes ayant une connaissance préalable sur la maladie, $18,80 \%$ ont reçu l'information d'un personnel de santé, 11,20\% par les médias et le plus fréquemment par un proche dans $32,40 \%$ des cas. Une femme pouvait avoir eu l'information par plusieurs de ces canaux.

On note qu'il n'y a pas de différence significative $\left(X^{2}=0,363 ; P=0,83\right)$ dans les réponses des femmes interviewées quel que soit la commune (Tableau 2). Le lieu 
d'habitation n'influence donc pas la connaissance de la maladie.

Par contre, pour les niveaux d'instruction, les tests statistiques montrent une différence très significative $\left(X^{2}=33,14\right.$; $P<0,001)$. Plus le niveau de scolarisation est élevé, plus les femmes interrogées ont une meilleure connaissance des fistules obstétricales (Tableau 3).

Les causes de la fistule obstétricale sont diverses. Parmi les femmes ayant déjà entendu parler de la maladie, $32,17 \%$ attribuent la survenue de cette pathologie aux accouchements difficiles, 6,99\% au nombre élevé d'accouchements, 10,49\% à l'excision, $10,49 \%$ à une vie aux mœurs légères. Le reste de ces femmes, soit $39,86 \%$, n'ont aucune idée sur l'étiologie.

En ce qui concerne la guérison des fistules obstétricales, $76,39 \%$ des femmes répondent oui tandis que $23,61 \%$ disent non (Tableau 4). Pour cet autre paramètre, la différence n'est pas significative entre les trois communes $\left(X^{2}=1,99 ; P=0,39\right)$.

Pour ce qui est de la méthode thérapeutique la mieux appropriée, $81,73 \%$ répondent que la médecine moderne est la plus adaptée pour solutionner le problème des fistules obstétricales tandis que 18,27\% fondent leur espoir sur la médecine traditionnelle (Tableau 5). La différence entre les communes n'est pas significative $\left(X^{2}=\right.$ $0,43 ; P=0,80)$. Ce qui traduit que le lieu d'habitation n'influence pas la qualité de la réponse.

\section{Quelques plantes utilisées en médecine traditionnelle contre la fistule obstétricale}

Suite aux enquêtes, une liste de 13 plantes médicinales potentiellement utilisables dans le traitement des fistules obstétricales a été dressée. Ces espèces sont reparties en 13 genres et neuf familles. La famille des
Lamiaceae est la plus représentée avec trois espèces, suivie de celles des Meliaceae, des Malvaceae et des Euphorbiaceae, avec deux espèces.

Les feuilles sont les organes les plus utilisés $(53,85 \%)$, suivies des écorces de tige $(46,15 \%)$. Le décocté est la forme médicamenteuse la plus utilisée $(76,92 \%)$ puis le broyage pour rendre l'organe en une pâte $(23,08 \%)$. La plupart des traitements sont administrées par voie anale $(61,54 \%)$ en lavement, voie vaginale sous forme de toilettes intimes $(46,15 \%)$ et la voie orale dans $30,77 \%$ des cas (Tableau 6).

Les fréquences de citation (FC) les plus élevées ont été obtenues pour Ocimum gratissimum et Alchornea cordifolia, avec $\mathrm{FC}=50 \%$ (Tableau 7). Ces espèces végétales sont suivies de Entandrophragma angolensis $(\mathrm{FC}=30 \%)$.

Ces trois plantes médicinales sont également les mêmes qui contribuent le plus aux différentes recettes médicamenteuses (Tableau 7). Les autres espèces végétales ont une contribution égale à $10 \%$.

On note que l'indice de consensus est de 1 pour Ocimum gratissimum et Alchornea cordifolia et très élevé avec Khaya senegalensis (IC $=0,71)$. Ceci montre qu'il y a plus d'accord autour de l'usage de ces espèces végétales. Autrement dit, il y a un grand nombre de personnes qui sont d'accord sur le rôle de ces plantes dans le traitement des fistules obstétricales. Elles sont suivies de Rhodognaphalon brevicupse et Tectona grandis, avec des IC $=0,36$ (Tableau 7 ). L'accord autour de l'utilisation des autres plantes médicinales est très faible $(\mathrm{IC}=0,17)$.

Quelques noms locaux de la maladie sont en malinké : Gninguènèdji-bana qui veut dire maladie de pipi, et en Abbey : Orogba qui signifie maladie de la honte. 


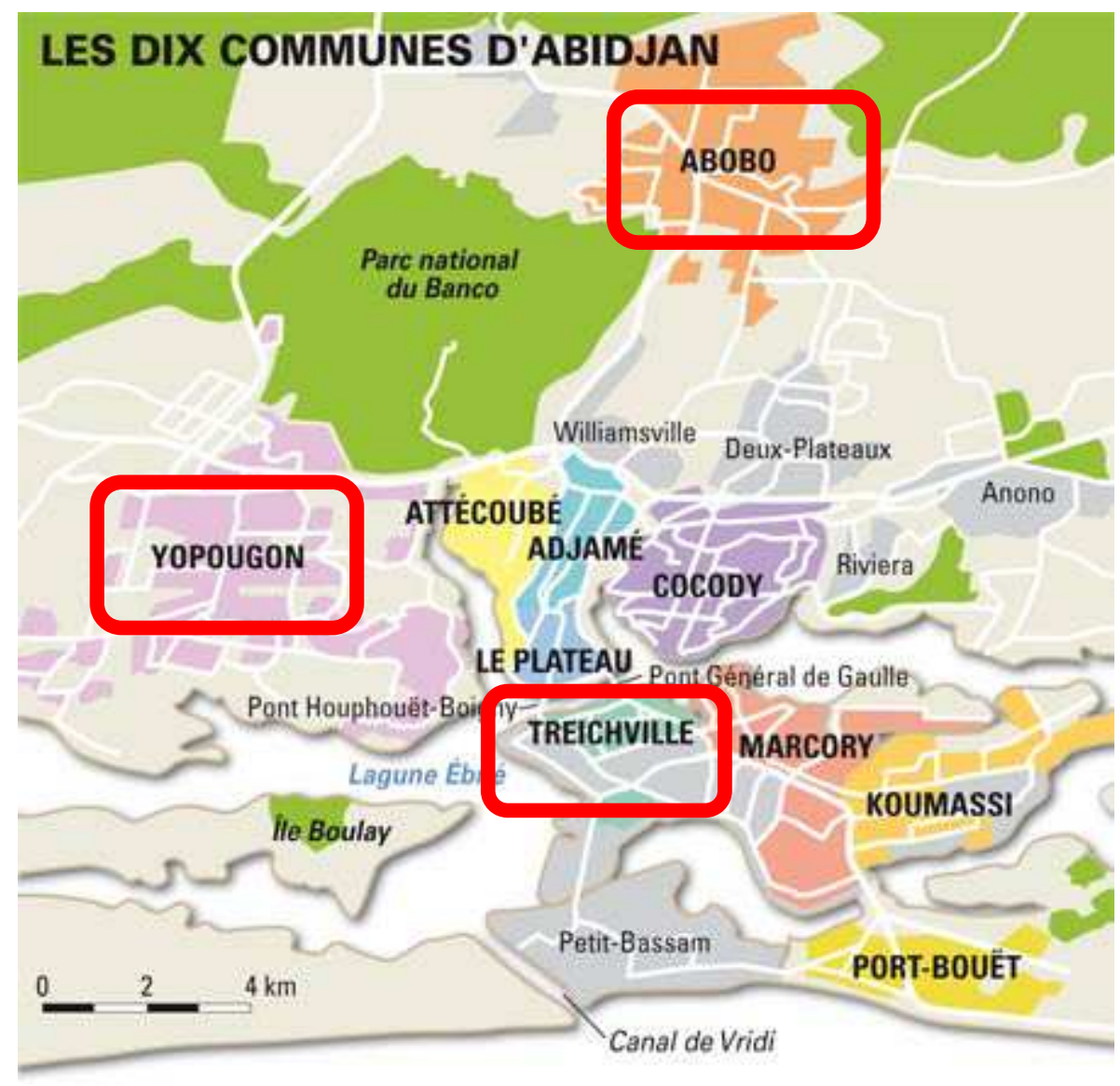

Zone d'étude

Figure 1 : Carte du district d'Abidjan, présentant les zones d'enquêtes.

Tableau 1 : Caractéristiques sociodémographiques des femmes interrogées.

\begin{tabular}{lcccc}
\hline Paramètres & \multicolumn{3}{c}{ Zone d'étude } & \multirow{2}{*}{ Moyenne } \\
\cline { 2 - 4 } & Abobo & Treichville & Yopougon & \\
\hline Effectif & 250 & 50 & 260 & 187 \\
Niveau d'étude & & & & \\
Analphabète & 93 & 19 & 62 & 58 \\
Primaire & 45 & 13 & 60 & 39 \\
Secondaire & 81 & 12 & 96 & 63 \\
Supérieur & 31 & 6 & 42 & 26 \\
\hline Classe d'âge & & & & \\
{$[18-30]$} & 102 & 10 & 77 & 63 \\
]30-45] & 124 & 26 & 126 & 92 \\
$>45$ & 24 & 14 & 57 & 32 \\
\hline
\end{tabular}




\begin{tabular}{lcccc}
\hline Situation social & & & & \\
Agent commercial & 8 & 0 & 15 & 8 \\
Artisan & 20 & 8 & 31 & 20 \\
Commerçante & 100 & 13 & 77 & 63 \\
Corps enseignant & 6 & 2 & 10 & 6 \\
Corps médical & 10 & 2 & 12 & 8 \\
Elève/ étudiante & 13 & 0 & 9 & 7 \\
Fille ménage & 4 & 9 & 9 & 7 \\
Fonctionnaire & 3 & 3 & 18 & 8 \\
Ménagère & 38 & 7 & 32 & 26 \\
Sans emploi & 48 & 6 & 47 & 34 \\
Statut matrimonial & & & & \\
Célibataire & 47 & 13 & 66 & 42 \\
Marié monogame & 174 & 31 & 168 & 124 \\
Marié polygame & 17 & 1 & 3 & 7 \\
Divorcé & 8 & 2 & 14 & 8 \\
Veuve & 4 & 3 & 9 & 5 \\
\hline
\end{tabular}

Tableau 2 : Niveau de connaissance des fistules obstétricales dans les différentes communes.

\begin{tabular}{|c|c|c|c|c|c|}
\hline \multirow[t]{2}{*}{ Communes } & \multicolumn{2}{|c|}{ Connaissance des fistules obstétricales } & \multicolumn{3}{|c|}{$\begin{array}{c}\text { Paramètres statistiques } \\
\text { de Khi-deux }\end{array}$} \\
\hline & Oui & Non & dl & $X^{2}$ & $P$ \\
\hline Abobo $(n=250)$ & 26,40 & 73,60 & \multirow{4}{*}{2} & \multirow{4}{*}{0,363} & \multirow{4}{*}{0,83} \\
\hline Treichville $(\mathrm{n}=50)$ & 28,00 & 72,00 & & & \\
\hline Yopougon $(n=260)$ & 24,62 & 75,38 & & & \\
\hline Total $(\mathrm{n}=560)$ & 25,71 & 74,29 & & & \\
\hline
\end{tabular}

Tableau 3 : Influence du niveau d'étude sur la connaissance des fistules obstétricales.

\begin{tabular}{|c|c|c|c|c|c|}
\hline \multirow[t]{2}{*}{ Niveau d'étude } & \multicolumn{2}{|c|}{$\begin{array}{c}\text { Connaissance des fistules } \\
\text { obstétricales }\end{array}$} & \multicolumn{3}{|c|}{$\begin{array}{c}\text { Paramètres } \\
\text { statistiques de Khi- } \\
\text { deux }\end{array}$} \\
\hline & OUI $^{1}$ & NON & dl & $X^{2}$ & $P$ \\
\hline Aucun $(n=174)$ & $7,47^{\mathrm{a}}$ & 92,52 & & & \\
\hline Primaire $(\mathrm{n}=118)$ & $16,94^{\mathrm{a}}$ & 83,05 & & & \\
\hline Secondaire $(n=189)$ & $34,92^{\mathrm{b}}$ & 65,07 & 3 & 33,14 & $<0,001$ \\
\hline Supérieur $(\mathrm{n}=79)$ & $56,96^{\mathrm{c}}$ & 43,03 & & & \\
\hline Total $(n=560)$ & 25,71 & 74,28 & & & \\
\hline
\end{tabular}


Tableau 4 : Etat de connaissance de la guérison des fistules.

\begin{tabular}{llllll}
\hline \multirow{2}{*}{ Communes } & \multicolumn{5}{c}{$\begin{array}{c}\text { Paramètres statistiques de } \\
\text { Khi-deux }\end{array}$} \\
\cline { 2 - 6 } & Oui & Non & dl & \multicolumn{1}{c}{$\boldsymbol{X}^{\mathbf{2}}$} & $\boldsymbol{P}$ \\
\hline Abobo $(\mathrm{n}=66)$ & 81,81 & 18,19 & & & \\
Treichville $(\mathrm{n}=14)$ & 71,42 & 28,58 & & & \\
Yopougon $(\mathrm{n}=64)$ & 71,87 & 28,13 & & & \\
Total $(\mathrm{n}=144)$ & 76,39 & 23,61 & & & \\
\hline \multicolumn{1}{c}{$\mathrm{n}=$ nombre, $\mathrm{dl}=$ degré de liberté, $X^{\mathbf{2}}=$ Khi-deux, $p=$ seuil. } & &
\end{tabular}

Tableau 5 : Méthodes thérapeutiques adaptées pour traiter la fistule obstétricale.

\begin{tabular}{|c|c|c|c|c|c|}
\hline \multirow[t]{2}{*}{ Communes } & \multicolumn{2}{|c|}{ Médecine adaptée } & \multicolumn{3}{|c|}{$\begin{array}{c}\text { Paramètres } \\
\text { statistiques de Khi- } \\
\text { deux }\end{array}$} \\
\hline & Moderne & Traditionnelle & dl & $X^{2}$ & $p$ \\
\hline Abobo $(n=56)$ & 83,93 & 16,07 & \multirow{4}{*}{2} & \multirow{4}{*}{0,43} & \multirow{4}{*}{0,80} \\
\hline Treichville $(\mathrm{n}=13)$ & 76,92 & 23,08 & & & \\
\hline Yopougon $(n=46)$ & 80,43 & 19,57 & & & \\
\hline Total $(\mathrm{n}=115)$ & 81,73 & 18,27 & & & \\
\hline
\end{tabular}

Tableau 6 : Plantes médicinales utilisées dans le traitement des fistules obstétricales.

\begin{tabular}{|c|c|c|c|c|}
\hline Espèces végétales & Famille & $\begin{array}{l}\text { Organe } \\
\text { utilisé }\end{array}$ & $\begin{array}{c}\text { Mode de } \\
\text { préparation }\end{array}$ & Posologie \\
\hline Abrus precatorius $\mathrm{L}$. & Fabaceae & Feuilles & Décoction & Boisson \\
\hline $\begin{array}{l}\text { Alchornea cordifolia (Schumach. \& } \\
\text { Thonn) Mull. Arg. }\end{array}$ & Euphorbiaceae & Feuilles & Décoction & $\begin{array}{l}\text { Toilette intime, } \\
\text { Bain de vapeur }\end{array}$ \\
\hline Bridelia ferruginea Benth & Phyllanthaceae & Feuilles & Ecraser & Lavement \\
\hline $\begin{array}{l}\text { Entandrophragma angolense (Welw. ex } \\
\text { C. DC.) C. DC. }\end{array}$ & Meliaceae & Ecorce de tige & Décoction & $\begin{array}{l}\text { Lavement, Boisson, } \\
\text { Toilette intime }\end{array}$ \\
\hline Heliotropium indicum $\mathrm{L}$. & Boraginaceae & Feuilles & Ecraser+piment & Lavement \\
\hline Hyptis pectinata (Linn.) Poit & Lamiaceae & Feuilles & Ecraser+piment & Lavement \\
\hline Khaya senegalensis (Desr.) A. Juss. & Meliaceae & Ecorce de tige & Décoction & Lavement \\
\hline Ocimum gratissimum $\mathrm{L}$. & Lamiaceae & Feuilles & Décoction & Bain de vapeur \\
\hline $\begin{array}{l}\text { Rhodognaphalon brevicuspe (Sprague) } \\
\text { Roberty }\end{array}$ & Malvaceae & Ecorce de tige & Décoction & $\begin{array}{l}\text { Lavement, toilette } \\
\text { intime }\end{array}$ \\
\hline Ricinodendron heudelotii (Baill.) Heckel & Euphorbiaceae & Ecorce de tige & Décoction & Boisson, Lavement \\
\hline Sterculia tragacantha Lindl. & Malvaceae & Ecorce de tige & Décoction & Lavement \\
\hline Tectona grandis L. f. & Lamiaceae & Feuilles & Décoction & $\begin{array}{l}\text { Boisson, Toilette } \\
\text { intime }\end{array}$ \\
\hline $\begin{array}{l}\text { Zanthoxylum zanthoxyloides (Lam.) } \\
\text { Zepern \& Timler }\end{array}$ & Rutaceae & Ecorce de tige & Décoction & Toilette intime \\
\hline
\end{tabular}


Tableau 7: Fréquence de citation, contribution et indice de consensus des espèces végétales inventoriées.

\begin{tabular}{lcccc}
\hline Espèces végétales & Familles & FC (\%) & Cpr (\%) & IC \\
\hline Abrus precatorius L. & Fabaceae & 10 & 10 & 0,17 \\
Alchornea cordifolia (Schumach. \& Thonn.) & Euphorbiaceae & 50 & 30 & 1 \\
Mull. Arg. & Phyllanthaceae & 10 & 10 & 0,17 \\
Bridelia ferruginea Benth. & Meliaceae & 30 & 20 & 0,17 \\
Entandrophragma angolense (Welw. ex C. & & & & \\
DC.) C. DC. & Boraginaceae & 10 & 10 & 0,17 \\
Heliotropium indicum L. & Lamiaceae & 10 & 10 & 0,17 \\
Hyptis pectinata (L.) Poit & Meliaceae & 20 & 10 & 0,71 \\
Khaya senegalensis (Desr.) A. Juss. & Lamiaceae & 50 & 30 & 1 \\
Ocimum gratissimum L. & Malvaceae & 20 & 10 & 0,36 \\
Rhodognaphalon brevicuspe (Sprague) & & & & \\
Roberty & Euphorbiaceae & 10 & 10 & 0,17 \\
Ricinodendron heudelotii (Baill.) Heckel & Malvaceae & 10 & 10 & 0,17 \\
Sterculia tragacantha Lindl. & Lamiaceae & 10 & 10 & 0,36 \\
Tectona grandis L. f. & Rutaceae & 10 & 10 & 0,17 \\
Zanthoxylum zanthoxyloides (Lam.) Zepern \& & & & & \\
Timler & & & \\
\hline
\end{tabular}

$\mathrm{FC}=$ fréquence de citation, $\mathrm{Cpr}=$ Contribution de chaque espèce aux recettes médicamenteuses, $\mathrm{IC}=$ indice de consensus

\section{DISCUSSION}

Les enquêtes sur la connaissance des fistules obstétricales et leur traitement par la médecine traditionnelle dans trois communes (Abobo, Treichville et Yopougon) du district d'Abidjan, ont montré que les femmes ont une faible connaissance des fistules obstétricales. Celles qui ont une connaissance de cette pathologie ont reçu l'information d'un proche dans $74,28 \%$ des cas. Ceci pourrait s'expliquer par les récentes campagnes de sensibilisation à l'échelle nationale. Plus de la moitié des femmes ayant une connaissance préalable des fistules obstétricales savent qu'elles sont guérissables. Cependant, le niveau d'étude constitue un facteur très déterminant dans la connaissance de la maladie. Les femmes avec un niveau d'instruction élevé sont plus informées sur la maladie et donc à même d'éviter sa survenue. De nombreux auteurs ont aussi identifié l'analphabétisme comme un facteur de risque pour développer une fistule obstétricale (Ijaiya et Aboveji, 2004 ; Wall et al., 2004 ; Tebeu et al., 2008). Selon Sanou et al. (2015), lorsqu'une femme est instruite, elle a plus de chance d'avoir une bonne culture sanitaire, ce qui la rend consciente de l'importance des soins obstétricaux de qualité et de l'adoption d'un meilleur comportement vis-à-vis de la médecine moderne.

Dans cette enquête, une question a été posée sur le statut matrimonial et socioécomonique des femmes interrogées. L'analyse des résultats montre que la majorité de ces femmes étaient mariées. Ce paramètre était important à prendre en compte. Selon Siborurema et al. (2012), plusieurs auteurs ont rapporté que le mariage est l'un des facteurs exposant aux fistules urogénitales d'origine obstétricales. Les résultats ont montré que les femmes enquêtées ont statut socioéconomique faible. Elles sont pour la plupart des commerçantes, des filles de ménages, des 
ménagères et sans emploi. Siborurema et al. (2012) ont rapporté une situation similaire au Rwanda. Or il existe une interrelation entre les conditions socio-économiques et les risques sanitaires pour les populations (Yonkeu et al., 2003). La santé s'améliore lorsque le revenu augmente, peu importe les habitudes de vie.

Pour une grande partie des femmes interrogées $(81,73 \%)$, la médecine moderne est le moyen le plus adaptée pour le traitement. Cependant, les 18,27\% restantes, ont cité la médecine traditionnelle comme une thérapie efficace, pour venir à bout des fistules obstétricales. Cette proportion n'est pas négligeable et encourage notamment les investigations sur les plantes médicinales.

Treize (13) plantes médicinales ont été recensées, elles sont reparties en 13 genres et neuf familles. A notre connaissance, l'usage de ces plantes médicinales dans le traitement des fistules obstétricales est rapporté ici pour la première fois, bien qu'elles aient été citées dans des travaux antérieurs d'ethnomédecine. Dans des enquêtes ethnobotaniques plus générales, les feuilles de Abrus precatorius sont utilisées par les peuples Abbey (Sud Côte d'Ivoire) pour faciliter l'accouchement (N'guessan et al., 2010 ; Lébri et al., 2015), et pour traiter les troubles gynéco-obstétriques (N'guessan et al., 2009). Parmi les 13 plantes inventoriées, Ocimum gratissimum et Alchornea cordifolia sont les deux espèces pour lesquelles il existe un consensus total (IC $=1$ ). Ce sont aussi les plantes dont l'implication est plus importante dans les recettes médicamenteuses. Ceci est certainement lié à la disponibilité de ces deux plantes sur les marchés d'Abidjan et dans les formations végétales environnantes. Ocimum gratissimum est une espèce cultivée. Un accord important (IC $=0,71)$ a été aussi obtenu autour de l'utilisation de Khaya senegalensis dans le traitement des fistules obstétricales. Ceci confirme le fait que cette plante est une espèce connue et largement utilisée en médecine traditionnelle en Côte
d'Ivoire (Koné et al., 2008) ainsi que dans d'autres pays en Afrique de l'Ouest (Dassou, 2016). Cette espèce figurait au nombre des plantes médicinales les plus vendues sur le marché d'Abomey-Calavi (Adomou et al., 2012). Sa faible contribution dans la préparation des recettes dans le district d'Abidjan pourrait s'expliquer par son origine géographique, les savanes du Nord de la Côte d'Ivoire.

La fistule obstétricale se caractérise par des déchirures douloureuses, plaies qui pourraient être infectées par diverses bactéries. Fort heureusement, la plupart des espèces végétales inventoriées sont connues pour leurs propriétés antimicrobienne, cicatrisante, anti-inflammatoire, etc. C'est le cas de Alchornea cordifolia qui est connue pour son activité antimicrobienne et cicatrisante des plaies (Traoré, 2004). L'activité antimicrobienne de Ocimum gratissimum a déjà été démontrée (Oussou et al., 2004 ; Adjou et al., 2013). Ces plantes médicinales pourraient être d'un intérêt dans le traitement des fistules obstétricales en Côte d'Ivoire.

\section{Conclusion}

L'enquête menée à Abobo, Treichville et Yopougon dans le district d'Abidjan, sur le niveau de connaissance des fistules obstétricales et leur traitement par la phytothérapie a montré que les fistules obstétricales sont peu connues des femmes interrogées dans ces communes. Le niveau de scolarisation est un facteur qui influence grandement la connaissance de cette affection. A côté de la médecine moderne, la médecine traditionnelle basée sur les plantes médicinales pourrait être un moyen pour le traitement de la maladie. Ainsi, les 13 plantes médicinales recensées seraient un champ d'investigation en vue de développer des thérapies alternatives pour le traitement des fistules obstétricales. 


\section{CONFLIT D'INTERETS}

Les auteurs ne déclarent aucun conflit d'intérêts.

\section{CONTRIBUTIONS DES AUTEURS}

SM-LL a rédigé le questionnaire, mené les enquêtes auprès des femmes, pris part à la rédaction et a approuvé la version finale du manuscrit; FHTB et $\mathrm{AB}$ ont procédé aux identifications des espèces végétales, pris part à la rédaction et la correction et approuvé la version finale du manuscrit; KY s'est occupé de tous les aspects statistiques (calcul de l'échantillonnage, analyses statistiques) depuis la confection du questionnaire jusqu'à l'analyse des résultats. Il a approuvé la version finale $\mathrm{du}$ manuscrit; MWK a développé le concept de l'étude, guidé la confection du questionnaire, supervisé les enquêtes sur le terrain, pris part à la rédaction et la correction et a approuvé la version finale du manuscrit.

\section{REMERCIEMENTS}

Les auteurs adressent leurs sincères remerciements au Centre National de Floristique (CNF) de l'Université Félix Houphouët-Boigny d'Abidjan, pour l'authentification des noms des espèces végétales. Ils remercient également toutes les femmes qui ont accepté de participer à cette étude.

\section{REFERENCES}

Adjou ES, Soumanou MM. 2013. Efficacité des extraits de plantes contre les

moisissures toxinogènes isolées da l'arachide. J. Appl. Biosci., 70: 5555-5566. DOI:

http://dx.doi.org/10.4314/jab.v70i1.98755 Adomou AC, Yedomonhan H, Djossa B, Legba SI, Oumorou M, Akoegninou A. 2012. Etude Ethnobotanique des plantes médicinales vendues dans le marché d'Abomey-Calavi au Bénin. Int. J. Biol.
Chem. Sci., 6(2): 745-772. DOI: http://dx.doi.org/10.4314/ijbcs.v6i2.18

Anderson FWJ, Mutchnick I, Kwawukume E Y, Danso KA, Klufio CA, Clinton Y, Yun, LL, Johnson TRB. 2007. Who Will be There When Women Deliver?: Assuring Retention of Obstetric Providers. Obstet. Gynecol., 110(5): 1012-1016. DOI: http://dx.doi.org/ 10.1097/01.AOG.0000287064.63051.1c

Blanchet A, Gotman A. 2007. L'enquête et ses méthodes : l'entretien. Nathan: Paris.

Bossart R. 2006. «En ville, chacun est dans son chacun » : une étude anthropologique sur l'importance des relations sociales en cas de maladie à Abidjan (Côte d'Ivoire). Peter Lang: Berne.

Bouvet JMP, Grimont ADP. 2001. Données de surveillance 1999 du centre national de référence des salmonella et shigella. Bull. Epidémiol. Hebdo., 12: 49-52. http://www.invs.sante.fr/beh/2001/12/beh _12_2001.pdf

Dassou GH. 2016. Diversité, ethnobotanique, écologie et statut de conservation des plantes utilisées en médecine vétérinaire traditionnelle au Bénin. Thèse de Doctorat en Botanique, Université d'Abomey-Calavi, Cotonou, p. 224.

Dassou GH, Ogni CA, Yedomonhan H, Tossou M, Dougnon JT, Akoegninou A. 2014. Diversité, usages vétérinaires et vulnérabilité des plantes médicinales au Nord-Bénin. Int. J. Biol. Chem. Sci., 8(1): 189-210.

DOI: http://dx.doi.org/10.4314/ijbcs.v8i1.18

Ijaiya MA, Aboyeji PA. 2004. Obstetric urogenital fistula: the Ilorin experience, Nigeria. West Afr. J. Med., 23(1): 7-9. DOI:

http://dx.doi.org/10.4314/wajm.v23i1.280 71

Kadjo B. 2014. Commune N'Zassa : Treichville, une intégration réussie- le témoignage des chefs de communautés. Notre Cité: Abidjan. 
Koné MW, Kamanzi Atindehou K, Traoré D. 2008. Use of ethnoveterinary medicinal plants in Northern Côte-d'Ivoire (West Africa). South Afr. J. Bot., 74(1): 76-84. DOI: http://dx.doi.org/ 10.1016/j.sajb.2007.08.015

Kouye P, Kabas G, Gbane M, Nda C. 2006. Étude fistules 2006 Étude SocioAnthropologique sur les Fistules Obstétricales en Côte d'Ivoire. DCPNSRPF/UNFPA: Abidjan.

Lebri M, Bahi C, Fofie NBY, Gnahoue G, Lagou SM, Achibat H, Yapi A, Zirihi GN, Coulibaly A, Hafid A, Khouili M. 2015. Analyse phytochimique et évaluation de la toxicité aiguë par voie orale chez des rats de l'extrait total aqueux des feuilles de Abrus precatorius Linn. (Fabaceae). Int. J. Biol. Chem. Sci., 9(3): 1470-1476. DOI : http://dx.doi.org/10.4314/ijbcs.v9i3.29

Marascuilo LA, Serlin RC. 1988. Statistical Methods for the Social and Behavioral Sciences. Freeman: New York.

Milliez J. 2012. La santé des femmes dans les pays en voie de développement. Bull. Acad. Natle Med., 196(8): 1509-1520. DOI: http://www.academiemedecine.fr/wpcontent/uploads/2013/10/2012.8.pdf

N'guessan K, Zihiri N, Boraud NKM. 2010. Etude ethnopharmacologique des plantes utilisées pour faciliter l'accouchement, en pays Abbey et Krobou, au Sud de la Côte d'Ivoire. Int. J. Biol Chem. Sci., 4(4): 1009-1010. DOI: http://dx.doi.org/ 10.4314/ijbcs.v4i4.63039

N'guessan K, Kouassi KE, Tiébré MS. 2009. Plantes utilisées dans le traitement des troubles gynéco-obstétriques par les peuples Abbey et Krobou d'Agboville, Côte d'Ivoire. Phytother., 7(5): 262-274. DOI: http://dx.doi.org/10.1007/s10298009-0411-x.

OMS (Organisation mondiale de la santé). 1991. Manuel d'Epidémiologie pour la
Gestion de la Santé au Niveau du District. OMS. Genève, Suisse ; 184.

OMS (Organisation Mondiale de la Santé). 2004. Profil du Système de Santé de la Côte d'Ivoire. Organisation Mondiale de la Santé: Genève.

OMS (Organisation mondiale de la santé). 2010. Côte d'Ivoire : Fiche d'Information des Statistiques Sanitaires 2010. Organisation mondiale de la santé: Genève.

Oussou KR, Kanko C, Guessend N, Yolou S, Dosso M, N'Guessan YT, Figueredo G, Chalchat J-C. 2004. Activités antibactériennes des huiles essentielles de trois plantes aromatiques de Côted'Ivoire. C. R. Chimie., 7(10-11): 10811086. DOI: http://dx.doi.org/10.1016/ j.crci.2003.12.034

Sanou SM, Sali BBA, Mabvouna BR, Douryang M, Teikeu TVV, Saah FMA, Ovaga ELM, Ausseil SM, Vittorio C, Russo G. 2015. Etude des connaissances, attitudes et pratiques en matière de réintégration sociale des femmes victimes de fistule obstétricale: région de l'Extrême-nord, Cameroun. Pan. Afr. Med. J., 20(1): 172. DOI: http://dx.doi.org/10.11604/pamj. 2015.20.172.5959

Siborurema L, Vyankandonder J, Utz B. 2012. Fistules Urogénitales d'origine Obstétricale: Aspects Épidémiologiques, Socioéconomiques, Cliniques et Thérapeutiques à l'Hôpital de Ruhengeri, Rwanda. Editions Universitaires Européennes: Paris.

Tebeu PM, Bernis DL, Boisrond L, Le Duc A, Mbassi AA, Rochat C-H. 2008. Connaissance, attitude et perception visà-vis des fistules obstétricales par les femmes camerounaises. Une enquête clinique conduite à Maroua, capitale de la province de l'extrême Nord du Cameroun. Progr Urol., 18(6): 379-389. 
DOI:

http://dx.doi.org/10.1016/ j.purol.2008.03.020

Traore M. 2004. Alchornea cordifolia. Schmach (Euphorbiaceae): revue des activités biologiques et l'étude de la toxicité. Thèse d'exercice, Université de Bamako, Bamako, p 107.

UNFPA (Fonds des Nations Unies pour la Population). 2003. The second meeting of the working group for the prevention and treatment of obstetric fistula, Addis Ababa, 30 october-1 november, 2002. UNFPA: New York. https://www.unfpa.org

UNFPA (Fonds des Nations Unies pour la Population) Population Council. 2013. Etude sur les fistules obstétricales. UNFPA: New York. https://www.unfpa.org

Vessereau A. 1992. Méthodes Statistiques en Biologie et en Agronomie. Tec et docLavoisier: Paris.

Wall LL, Karshima JA, Kirschner C, Arrowsmith SD. 2004. The obstetric vesicovaginal fistula: characteristics of 899 patients from Jos, Nigeria. Am. J.
Obstet. Gynecol., 190: 1011- 1019. DOI: http://dx.doi.org/10.1016/j.ajog.2004.02.0 07

Wall LL. 2006. Obstetric vesicovaginal fistula as an international public-health problem. Lancet, 368(9542): 1201-1209. DOI: http://dx.doi.org/10.1016/S01406736(06)69476-2

Wall LL. 2012. Preventing obstetric fistulas in low-resource countries: insights from a Haddon matrix. Obstet Gynecol Surv., 67(2): 111-21. DOI: http://dx.doi.org/10.1097/OGX.0b013e31 82438788

Xavier JF, Randrianirinarison JC, Ravelojoana M. 2007. Analyse de la Situation de Base sur l'Ampleur et les Impacts des Fistules obstétricales à Madagascar. UNFPA: New York.

Yonkeu S, Maïga AH, Wethé J, Mampouya M, Maga GP. 2003. Conditions socioéconomiques des populations et risques de maladies: Le bassin versant $d u$ barrage de Yitenga au Burkina Faso. VertigO., $\quad 4(1)$. $\quad$ DOI : http://dx.doi.org/10.4000/vertigo.4778 